\title{
RESEARCH
}

Open Access

\section{The EMIF-AD Multimodal Biomarker Discovery study: design, methods and cohort characteristics}

Isabelle Bos ${ }^{1,33^{*}}$, Stephanie Vos ${ }^{1}$, Rik Vandenberghe ${ }^{2,3}$, Philip Scheltens ${ }^{4}$, Sebastiaan Engelborghs ${ }^{5,6,7}$, Giovanni Frisoni ${ }^{8,9}$, José Luis Molinuevo ${ }^{10,11}$, Anders Wallin ${ }^{12}$, Alberto Lleó ${ }^{13}$, Julius Popp ${ }^{14,15}$, Pablo Martinez-Lage ${ }^{16}$, Alison Baird ${ }^{17}$, Richard Dobson ${ }^{18,19,20,21}$, Cristina Legido-Quigley ${ }^{18}$, Kristel Sleegers ${ }^{22,23}$, Christine Van Broeckhoven ${ }^{22,23}$, Lars Bertram 24,25,26, Mara ten Kate ${ }^{4}$, Frederik Barkhof ${ }^{27}$, Henrik Zetterberg 28,29,30,31, Simon Lovestone ${ }^{17}$, Johannes Streffer ${ }^{32}$ and Pieter Jelle Visser ${ }^{1,4}$

\footnotetext{
Abstract

Background: There is an urgent need for novel, noninvasive biomarkers to diagnose Alzheimer's disease (AD) in the predementia stages and to predict the rate of decline. Therefore, we set up the European Medical Information Framework for Alzheimer's Disease Multimodal Biomarker Discovery (EMIF-AD MBD) study. In this report we describe the design of the study, the methods used and the characteristics of the participants.

Methods: Participants were selected from existing prospective multicenter and single-center European studies. Inclusion criteria were having normal cognition (NC) or a diagnosis of mild cognitive impairment (MCI) or AD-type dementia at baseline, age above 50 years, known amyloid-beta (A $\beta$ ) status, availability of cognitive test results and at least two of the following materials: plasma, DNA, magnetic resonance imaging (MRI) or cerebrospinal fluid (CSF). Targeted and untargeted metabolomic and proteomic analyses were performed in plasma, and targeted and untargeted proteomics were performed in CSF. Genome-wide SNP genotyping, next-generation sequencing and methylation profiling were conducted in DNA. Visual rating and volumetric measures were assessed on MRI. Baseline characteristics were analyzed using ANOVA or chi-square, rate of decline analyzed by linear mixed modeling.

Results: We included 1221 individuals (NC $n=492, \mathrm{MCl} n=527$, AD-type dementia $n=202$ ) with a mean age of 67.9 (SD 8.3) years. The percentage A 3 + was $26 \%$ in the $\mathrm{NC}, 58 \%$ in the $\mathrm{MCl}$, and $87 \%$ in the AD-type dementia groups. Plasma samples were available for 1189 (97\%) subjects, DNA samples for 929 (76\%) subjects, MRI scans for 862 (71\%) subjects and CSF samples for 767 (63\%) subjects. For 759 (62\%) individuals, clinical follow-up data were available. In each diagnostic group, the APOE $\varepsilon 4$ allele was more frequent amongst $A \beta+$ individuals $(p<0.001)$. Only in $\mathrm{MCl}$ was there a difference in baseline Mini Mental State Examination (MMSE) score between the A groups $(p<0.001)$. A $\beta+$ had a faster rate of decline on the MMSE during follow-up in the NC $(p<0.001)$ and $\mathrm{MCI}(p<0.001)$ groups.

\footnotetext{
* Correspondence: isabelle.bos@maastrichtuniversity.nl

'Alzheimer Centrum Limburg, Maastricht University, Maastricht, the Netherlands

${ }^{33}$ Department of Psychiatry \& Neuropsychology, School for Mental Health

and Neuroscience, Alzheimer Center Limburg, Maastricht University,

Universiteitssingel 40, Box 34, P.O. Box 616,6200, MD, Maastricht, the

Netherlands

Full list of author information is available at the end of the article
}

(c) The Author(s). 2018 Open Access This article is distributed under the terms of the Creative Commons Attribution 4.0 International License (http://creativecommons.org/licenses/by/4.0/), which permits unrestricted use, distribution, and reproduction in any medium, provided you give appropriate credit to the original author(s) and the source, provide a link to the Creative Commons license, and indicate if changes were made. The Creative Commons Public Domain Dedication waiver (http://creativecommons.org/publicdomain/zero/1.0/) applies to the data made available in this article, unless otherwise stated. 
(Continued from previous page)

Conclusions: The characteristics of this large cohort of elderly subjects at various cognitive stages confirm the central roles of $A \beta$ and $A P O E \varepsilon 4$ in AD pathogenesis. The results of the multimodal analyses will provide new insights into underlying mechanisms and facilitate the discovery of new diagnostic and prognostic AD biomarkers. All researchers can apply for access to the EMIF-AD MBD data by submitting a research proposal via the EMIF-AD Catalog.

Keywords: Alzheimer's disease, Biomarkers, Multimodal, Proteomics, Genomics, Metabolomics, Plasma, Magnetic resonance imaging, DNA, Cerebrospinal fluid,

\section{Background}

Over the last decade great progress has been made in diagnosing Alzheimer's disease (AD) at an early disease stage, including before the onset of dementia $[1,2]$. The biomarkers amyloid-beta $(A \beta)$ and tau in cerebrospinal fluid (CSF) or amyloid and tau load via positron emission tomography (PET) have become indispensable in the AD research field, especially as part of clinical trials for disease modification and secondary prevention [3-6]. Nonetheless, a better understanding of the underlying pathophysiological disease mechanisms as well as the discovery of diagnostic and prognostic markers that are inexpensive and minimally invasive to obtain would enhance the development of therapeutic interventions.

Currently, CSF and PET biomarkers are commonly used for the early diagnosis and prognosis of AD [7-9]. However PET imaging is fairly expensive and not universally available and the procedure for obtaining a PET scan as well as CSF data are relatively invasive. Given this, complementing these highly specific biomarker modalities with markers in more readily accessible biofluids would mark an important step forward. Consequently, many initiatives have been undertaken to discover and validate blood-based biomarkers for $\mathrm{AD}$ pathology [10, 11], but so far results have been limited, due to small sample sizes, single modality analyses or other methodological issues [12]. One critical issue so far has been the design (comparing individuals with AD-type dementia with controls), which made the studies unsuitable for discovery of markers for the preclinical disease phase. To seek markers for the preclinical phase, a more sensitive and gradual approach has been proposed, described as the "endophenotype approach" where discovery is predicted on a measure of pathology [13]. Therefore, we designed the current study to enhance blood-based biomarker discovery by performing a series of omics techniques (e.g., proteomics, metabolomics, genomics) in a large cohort across the $\mathrm{AD}$ clinical disease spectrum, using an endophenotype approach.

This study was performed as a part of the European Medical Information Framework for Alzheimer's disease (EMIF-AD; http://www.emif.eu). Funded through the Innovative Medicines Initiative (IMI), the EMIF project was established to facilitate the process of reusing and combining existing healthcare data with a focus on two therapeutic areas in the first instance: metabolic diseases and $\mathrm{AD}$. One of the main aims of the EMIF-AD project is to accelerate the discovery of novel diagnostic and prognostic biomarkers for $\mathrm{AD}$ and to unravel the underlying pathophysiological mechanisms, using existing data and existing samples, that would otherwise be inaccessible to research beyond the project teams responsible for the collection. In this report, we will describe the set-up of the EMIF-AD Multimodal Biomarker Discovery (EMIF-AD MBD) study, the methods as well as the characteristics of the included subjects. The results of the single and multimodal analyses will be described in future publications.

\section{Methods}

\section{General outline}

In the EMIF-AD MBD study we retrospectively combined and reused clinical data, samples and scans that had already been collected as part of existing prospective cohort studies. We aimed to include a total of 1000 subjects across the clinical AD spectrum: 400 subjects with normal cognition (NC), 400 subjects with mild cognitive impairment (MCI) and 200 subjects with mild AD-type dementia. To create a balanced design in terms of progression and to enable endophenotype designed biomarker studies, we intended to include $50 \% \mathrm{~A} \beta$-positive $(\mathrm{A} \beta+)$ individuals and $50 \%$ $A \beta$-negative $(A \beta-)$ individuals in the groups with $N C$ and MCI. To conduct multimodal analyses, we initially aimed to include subjects who had material from MRI, plasma, DNA and CSF. Later, we adjusted this to subjects with material available in at least two of the modalities listed.

\section{Selection of cohorts}

We used the EMIF-AD Catalog (https://emif-catalogue.eu), established as part of the objective of the EMIF which seeks to enable the finding, assessment and reutilization of preexisting data. The EMIF-AD Catalog contains metadata about European AD cohorts, enabling the selection of studies that included subjects who, in this instance, met the following inclusion criteria: data on $A \beta$ status, measured in CSF or by amyloid positron emission tomography (PET); age above 50 years at baseline; and availability of MRI scans, plasma and DNA samples. We identified 16 suitable cohorts. Two cohorts declined due 
to other research interests. Three cohorts were interested to collaborate, but unable because of legal and/or ethical restrictions, or unavailability of sufficient sample volumes. The 11 selected cohorts included three multicenter studies-EDAR $(n=204)$ [14], PharmaCog $(n=147)$ [15] and DESCRIPA $(n=29)$ [16] —and eight single centers: Antwerp $(n=149)$ [17], Amsterdam $(n=172)$ [18], Barcelona Sant Pau $(n=45)$ [19], Barcelona IDIBAPS $(n=120)$ [20], Leuven $(n=180)$ [21], San Sebastian GAP $(n=40)$ [22], Gothenburg $(n=95)$ [23] and Lausanne $(n=40)$ [24]. Of these 11 cohorts, DESCRIPA, EDAR, PharmaCog, Amsterdam, Antwerp and Gothenburg were linked to partners in the EMIF-AD, while the other five cohorts participated as affiliated data providers (ADP). All cohorts (e.g., partners and ADP) signed a material transfer agreement. The ADP also agreed to the EMIF project agreement. Study managers from each cohort selected the subjects based on the following criteria: age above 50 years at baseline; availability of $A \beta$ status at baseline measured in CSF or via PET; availability of neuropsychological and clinical data; availability of at least two of the following materials: MRI scan, plasma sample, DNA samples or CSF sample; and absence of neurological, psychiatric or somatic disorders that could cause cognitive impairment. The local medical ethical committee in each center approved the study. Subjects had already provided written informed consent at the time of inclusion in the cohort for use of data, samples and scans. Figure 1 shows a timeline of the different events in establishing this cohort, from the search in the EMIF Catalog to the wet-lab analyses.

\section{Baseline diagnoses}

In all cohorts, the definition for $\mathrm{NC}$ was a normal performance on neuropsychological assessment (within 1.5 SD of the average for age, gender and education). Five cohorts also used a score of 0 on the Clinical Dementia Rating
(CDR) [25] and a single cohort used a cutoff value $<3$ on the Global Deterioration Scale [26] to determine NC. Diagnosis of MCI was made according to the criteria of Petersen [27] in nine cohorts. Two cohorts used the Winblad et al. criteria [28] to diagnose MCI. All cohorts used the National Institute of Neurological and Communicative Disorders and Stroke-Alzheimer's Disease and Related Disorders Association criteria (NINCDS-ADRDA) criteria [29] to diagnose AD-type dementia. Additional file 1: Table S1 presents the diagnostic criteria used per center.

\section{Clinical data}

All cohorts were asked to contribute available data on demographics, clinical information, neuropsychological testing and $A \beta$ status, as presented in Table 1. Medication use and comorbidities were classified into a number of categories, for which we created dichotomous variables (Table 1).

Cognitive data were collected in all cohorts. The cognitive tests used varied across centers. Only the Mini Mental State Examination (MMSE) was administered in all centers and was available for nearly all subjects $(n=1216)$. We requested at least one test from the following cognitive domains: memory, language, attention, executive functioning and visuoconstruction [16]. For each cognitive domain, we selected a primary test (Table 1 ). If the preferred tests were not available, we selected an alternative priority test from the same cognitive domain. Additional file 2: Table S2 provides an overview of the different tests used for each cognitive domain. For each test, we requested the raw scores and, if available, $z$-scores calculated based on local normative data. If local normative data were unavailable, we calculated $z$-scores based on published normative data from healthy controls for that test. Per cognitive domain, we combined $z$-scores which

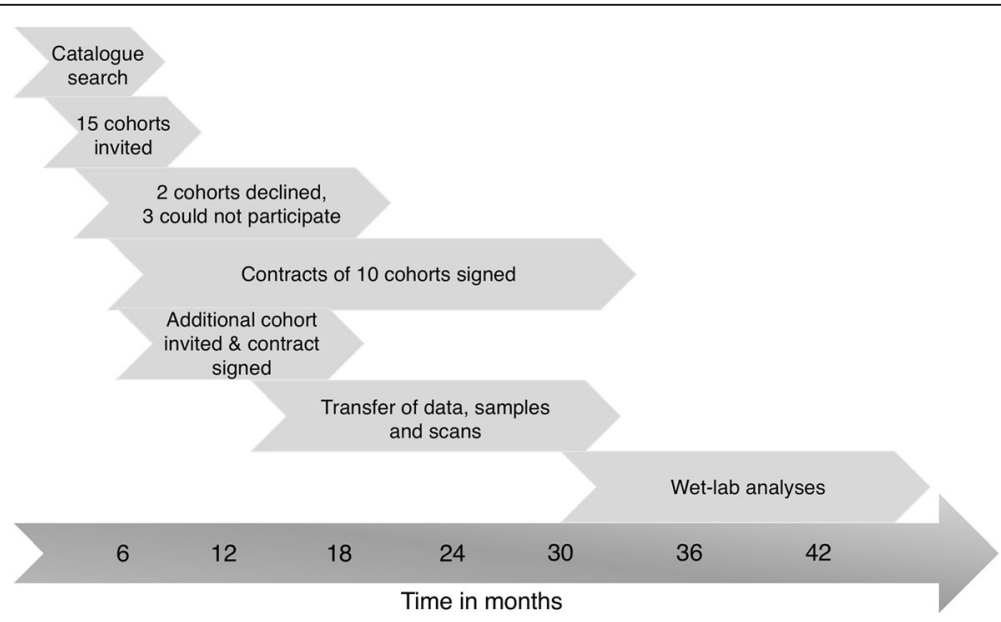

Fig. 1 Timeline of events related to the EMIF-AD MBD study 
Table 1 Clinical dataset

\begin{tabular}{|c|}
\hline Demographics \\
\hline Age \\
\hline Gender \\
\hline Years of education \\
\hline Clinical information \\
\hline Diagnosis \\
\hline Medication use \\
\hline Cardiovascular medication \\
\hline Dementia medication \\
\hline Hormonal medication \\
\hline Psychopharmaceuticals \\
\hline Other medication \\
\hline Comorbidities \\
\hline Cardiovascular disorders \\
\hline Cardiovascular risk factors \\
\hline Cerebrovascular disorders \\
\hline Endocrine disorders \\
\hline Neurological disorders \\
\hline Other cardiac disorders \\
\hline Psychiatric disorders \\
\hline Somatic disorders \\
\hline Family history of dementia \\
\hline First-degree relatives \\
\hline Second-degree relatives \\
\hline Functional impairment rating \\
\hline Neuropsychological tests \\
\hline Memory, preferred test: AVLT \\
\hline Language, preferred test: animal fluency \\
\hline Attention, preferred test: Trail Making Test A \\
\hline Executive functioning, preferred test: Trail Making Test B \\
\hline Visuoconstruction, preferred test: Rey complex figure copy \\
\hline$A \beta$ measure $^{a}$ \\
\hline CSF $A \beta_{42}$ value and local cutoff point \\
\hline Amyloid PET SUV and local cutoff point \\
\hline
\end{tabular}

${ }^{a} A t$ least one $A \beta$ measure

$A \beta$ amyloid-beta, AVLT Auditory Verbal Learning Test, CSF cerebrospinal fluid, PET positron emission tomography, SUV standardized uptake value

we used as a continuous variable, and we used a cutoff value of $z$-score $<-1.5$ to define abnormality.

Clinical data were harmonized, pooled and stored on an online data platform using tranSMART [30], now enriched for dementia research purposes through the EMIF-AD project.

\section{Plasma analyses}

Initially, the minimum requested amount of plasma was $0.7 \mathrm{ml}$. If available, another $0.7 \mathrm{ml}$ was requested to conduct additional analyses in a subgroup of subjects. In some cases, only $0.5 \mathrm{ml}$ was available. Prior to the analyses, samples were checked visually for consistency and volume. Results of analyses were also quality checked by inspecting patterns of outliers, and excluding consistent outliers across analyses. Analyses conducted on these samples include: targeted analyses of plasma proteins identified previously [31] and confirmed in at least one replication study, a panel of complement proteins nominated because of increasing evidence from genomics of the role of innate immunity in $\mathrm{AD}$ and analysis of plasma neurofilament light (NFL) chain; untargeted proteomic analyses using aptamer capture approaches; and untargeted and targeted metabolic analyses using a 883-metabolite panel for the targeted assay.

\section{Genetic analyses}

A total amount of $2.6 \mu \mathrm{g}$ DNA or $1 \mathrm{ml}$ whole blood, from which DNA was to be extracted, was requested for the genetic analyses. After performing routine quality checks on extracted DNA (e.g., agarose gel electrophoresis, determination of A260/280 and A260/230 ratios, PicoGreen quantification), we performed three types of assessments on each sample passing quality control: genome-wide SNP genotyping (Global Screening Array; Illumina, Inc.), genome-wide DNA methylation profiling (Infinium MethylationEPIC BeadChip; Illumina, Inc.) and whole exome sequencing.

\section{CSF analyses}

The requested amount of CSF to conduct all planned analyses was $0.4 \mathrm{ml}$, which was used for untargeted proteomic and peptidomic analyses, and a number of targeted analyses measuring concentrations of $A \beta_{38}, A \beta_{40}, A \beta_{42}$, $A \beta_{42 / 40}$, YKL-40, NFL and neurogranin (Ng). Prior to the analyses, CSF samples were checked visually for volume and absence of blood contamination.

\section{MRI analyses}

MRI scans were assembled centrally, quality checked and assessed visually by a single rater. T1-weighted and, when available, FLAIR and/or T2\%/SWI images were used for qualitative visual rating, including medial temporal lobe atrophy [32], global cortical atrophy [33], white matter hyperintensities [34] and microbleeds (defined as small $(<$ $10 \mathrm{~mm}$ ) round foci of hypointense signal in brain parenchyma). 3D T1 scans were uploaded to the Neurgrid platform (https://neugrid4you.eu) [35] for storage and automated quantitative analyses. Volumetric analysis included assessment of hippocampal and whole brain volume and cortical thickness.

\section{Amyloid classification}

$A \beta$ status was defined by the CSF $A \beta_{42 / 40}$ of the central analyses, using a cutoff value of $<0.061$ to determine 
abnormality $(n=770)$. The cutoff value for the $A \beta_{42 / 40}$ ratio was determined based on mixture model analyses comparing the $\mathrm{NC}$ and $\mathrm{AD}$ groups in this dataset. When no CSF was contributed for central analyses, the local CSF $A \beta_{42}$ value $(n=271)$ or the standardized uptake value ratio (SUVR) on an amyloid PET scan $(n=180)$ with local cutoff values to determine abnormality were used (Additional file 3: Table S3).

\section{Statistical analyses}

Baseline characteristics were compared between groups using ANOVA for continuous variables and chi-square for categorical variables. General linear mixed models with random intercepts and slopes by study were used to examine the influence of $A \beta$ status on MMSE performance and decline over time, adjusted for age, gender and years of education. Missing values for APOE genotype $(n=12)$ and years of education $(n=105)$ were imputed using regression within study with at least two significant predictors (i.e., age, gender, MMSE, etc.). Statistical analyses were performed using R Statistical Software (version 3.3.3) and SPSS (version 24), with significance defined as $p<0.05$.

\section{Results}

We initially sought to identify 1000 individuals with data available in all modalities. However, because not all studies could contribute data for all modalities, we included more participants to meet the aimed number of individuals for each modality. In total, 1221 subjects were included in the study, with a mean age of 67.9 (SD 8.3) years. Six hundred and sixty-seven (54\%) were female and the average education level was 11.7 (SD 4.1) years. At baseline, 492 (40\%) subjects had NC, 527 (43\%) subjects had a diagnosis of MCI and $202(17 \%)$ subjects had a clinical diagnosis of AD-type dementia. For 758 (62\%) individuals there were follow-up data available (e.g., at least a clinical diagnosis or MMSE at follow-up): 217 (44\%) NC subjects, 398 (76\%) MCI subjects and 143 (71\%) demented subjects. The average follow-up time for all 758 individuals was 2.3 (SD 1.2) years. Per diagnostic groups, the average clinical follow-up time was: NC 2.4 (SD 0.9) years, MCI 2.2 (SD 1.3) years and $\mathrm{AD} 2.2$ (SD 1.4) years.

Table 2 presents the baseline characteristics of the sample by $A \beta$ status and by baseline diagnosis. In the $\mathrm{NC}$ and MCI groups, the $\mathrm{A} \beta+$ subjects were older than the $\mathrm{A} \beta$ - subjects $(\mathrm{NC}, p=0.002 ; \mathrm{MCI}, p<0.001)$. In all diagnostic groups, $A \beta+$ subjects were more likely to be an APOE $\varepsilon 4$ carrier (all $p<0.001$ ). In the MCI subjects only, there was a difference in baseline MMSE score between the $\mathrm{A} \beta$ groups $(p=0.001)$. Regarding cognitive domains, we found differences in memory $(p<0.001)$ and executive functioning $(p=0.042) z$-scores in individuals with $\mathrm{MCI}$. In individuals with AD-type dementia we found that $\mathrm{A} \beta+$ individuals performed worse on an executive functioning task $(p=0.013)$.

Table 3 presents the number of subjects per modality by diagnostic category. Plasma samples were contributed for 1189 (97\%) subjects, DNA for 929 (76\%) subjects, MRI scans for $862(71 \%)$ subjects and CSF for $770(63 \%)$ subjects. There were $482(40 \%)$ subjects who contributed material in all modalities. Of this subsample, 89 (18\%) subjects had NC, 318 (66\%) subjects MCI and 75 (16\%) subjects had a diagnosis of AD-type dementia at baseline.

Table 2 Baseline characteristics by clinical diagnosis and $A \beta$ status

\begin{tabular}{|c|c|c|c|c|c|c|c|}
\hline & & \multicolumn{2}{|c|}{ Normal cognition } & \multicolumn{2}{|l|}{$\mathrm{MCl}$} & \multicolumn{2}{|c|}{ AD-type dementia } \\
\hline & & $A \beta-$ & $A \beta+$ & $A \beta-$ & $A \beta+$ & $A \beta-$ & $A \beta+$ \\
\hline & Total $n$ & $n=365$ & $n=127$ & $n=220$ & $n=307$ & $n=27$ & $n=175$ \\
\hline Age (years) & 1221 & $64.4(7.6)$ & $66.9(7.9)^{* *}$ & $68.3(8.2)$ & $70.7(7.4)^{* * *}$ & $73.0(8.4)$ & $69.9(8.8)$ \\
\hline Female, $n$ & 1221 & $203(56)$ & $66(52)$ & $108(49)$ & $172(56)$ & $12(44)$ & $96(55)$ \\
\hline Education (years) & 1221 & $13.5(3.7)$ & $12.7(4.0)^{*}$ & $10.6(3.8)$ & $10.8(3.7)$ & $8.5(4.4)$ & $10.6(3.8)^{* *}$ \\
\hline APOE $\varepsilon 4$ carrier, $n$ & 1221 & $122(33)$ & $76(60)^{* * *}$ & $46(21)$ & $200(65)^{* * *}$ & $7(26)$ & $114(65)^{* * *}$ \\
\hline Mean follow-up time (years) & 758 & $2.3(0.8)$ & $2.5(1.1)$ & $2.2(1.3)$ & $2.2(1.3)$ & $1.7(0.9)$ & $2.2(1.4)$ \\
\hline MMSE score & 1215 & $28.9(1.1)$ & $28.8(1.2)$ & $27.0(2.3)$ & $25.9(2.7)^{* * *}$ & $21.5(5.4)$ & $21.7(4.6)$ \\
\hline Memory delayed z-score & 1049 & $0.1(1.1)$ & $0.0(1.2)$ & $-0.9(1.3)$ & $-1.4(1.4)^{* * *}$ & $-2.2(1.2)$ & $-2.4(1.1)$ \\
\hline Language $z$-score & 1181 & $-0.2(1.0)$ & $-0.1(1.0)$ & $-0.7(1.2)$ & $-1.0(2.0)$ & $-1.9(1.2)$ & $-2.3(2.4)$ \\
\hline Attention z-score & 1128 & $0.3(1.1)$ & $0.2(0.9)$ & $-1.0(1.8)$ & $-1.0(1.8)$ & $-2.1(2.5)$ & $-2.1(2.0)$ \\
\hline Executive functioning $z$-score & 976 & $0.3(1.1)$ & $0.1(1.1)$ & $-0.9(1.9)$ & $-1.4(2.1)^{*}$ & $-1.2(2.5)$ & $-3.4(2.8)^{*}$ \\
\hline Visuoconstruction z-score & 664 & $0.2(1.4)$ & $0.2(0.8)$ & $-0.3(1.7)$ & $-0.4(1.8)$ & $-2.1(2.4)$ & $-1.3(2.0)$ \\
\hline
\end{tabular}

Results are mean (standard deviation) for continuous variables or frequency (\%) for dichotomous variables

$A \beta$ amyloid-beta, $A D$ Alzheimer's disease, $A P O E$ apolipoprotein $\mathrm{E}, \mathrm{MCl}$ mild cognitive impairment, MMSE Mini Mental State Examination

${ }^{*} p<0.05$ in comparison to $A \beta$ - group

${ }^{* *} p<0.01$ in comparison to $A \beta$ - group

${ }^{* * *} p<0.001$ in comparison to $A \beta$ - group 
Table 3 Number of subjects from different cohorts for each modality by diagnosis

\begin{tabular}{|c|c|c|c|c|c|c|}
\hline Cohort & Diagnosis & Clinical data & Plasma & DNA & MRI & CSF \\
\hline \multirow[t]{3}{*}{ Amsterdam } & NC & 30 & 29 & 26 & 30 & 30 \\
\hline & $\mathrm{MCl}$ & 82 & 80 & 68 & 82 & 82 \\
\hline & AD-type dementia & 60 & 60 & 53 & 60 & 60 \\
\hline \multirow[t]{2}{*}{ Antwerp } & $\mathrm{MCl}$ & 103 & 100 & 101 & 50 & 103 \\
\hline & AD-type dementia & 46 & 47 & 46 & 0 & 46 \\
\hline \multirow[t]{2}{*}{ DESCRIPA } & NC & 12 & 12 & 8 & 5 & 12 \\
\hline & $\mathrm{MCl}$ & 17 & 17 & 12 & 9 & 17 \\
\hline \multirow[t]{3}{*}{ EDAR } & NC & 48 & 47 & 42 & 14 & 47 \\
\hline & $\mathrm{MCl}$ & 77 & 75 & 65 & 24 & 75 \\
\hline & AD-type dementia & 79 & 78 & 69 & 19 & 76 \\
\hline GAP & NC & 40 & 40 & 40 & 38 & 40 \\
\hline \multirow[t]{2}{*}{ Gothenburg } & NC & 49 & 48 & - & 48 & - \\
\hline & $\mathrm{MCl}$ & 46 & 44 & - & 46 & - \\
\hline \multirow[t]{3}{*}{ IDIBAPS } & NC & 76 & 77 & - & 40 & - \\
\hline & $\mathrm{MCl}$ & 27 & 27 & - & 14 & - \\
\hline & AD-type dementia & 17 & 16 & - & 14 & - \\
\hline \multirow[t]{2}{*}{ Lausanne } & NC & 12 & 12 & 12 & 12 & 12 \\
\hline & $\mathrm{MCl}$ & 28 & 28 & 28 & 27 & 28 \\
\hline Leuven & NC & 180 & 163 & 168 & 179 & - \\
\hline PharmaCog & $\mathrm{MCl}$ & 147 & 144 & 146 & 147 & 147 \\
\hline Sant Pau & NC & 45 & 45 & 45 & - & - \\
\hline \multirow[t]{4}{*}{ Total } & NC & 492 & 473 & 341 & 366 & 141 \\
\hline & $\mathrm{MCl}$ & 527 & 515 & 420 & 399 & 452 \\
\hline & AD-type dementia & 202 & 201 & 168 & 100 & 182 \\
\hline & Overall & 1221 & 1189 & 929 & 865 & 775 \\
\hline
\end{tabular}

CSF cerebrospinal fluid, DESCRIPA development of screening guidelines and clinical criteria for predementia Alzheimer's disease, EDAR beta amyloid oligomers in the early diagnosis of $A D$ and as a marker for treatment reponse, GAP gipuzkoa Alzheimer project, IDIBAPS institut d'investigacions biomèdiques August $\mathrm{Pi}$ i Sunyer, $\mathrm{MCl}$ mild cognitive impairment, $\mathrm{MRI}$ magnetic resonance imaging, NC normal cognition

Table 4 and Fig. 2 show the effect of A $\beta$ on MMSE scores over time for each diagnostic group, adjusted for demographics. At baseline, there is only a difference in MMSE for the MCI group $(p<0.001)$. In the NC and MCI groups, the $\mathrm{A} \beta+$ individuals in the $\mathrm{NC}$ and $\mathrm{MCI}$ groups

Table 4 Effect of A $\beta$ on MMSE scores over time by diagnostic group

\begin{tabular}{llllll}
\hline Diagnosis & $n$ & Baseline & $p$ value & Slope & $p$ value \\
\hline NC & 482 & $-0.35 \pm 0.20$ & 0.170 & $-0.60 \pm 0.13$ & $<0.001$ \\
MCl & 459 & $-1.56 \pm 0.24$ & $<0.001$ & $-0.60 \pm 0.14$ & $<0.001$ \\
AD dementia & 162 & $-0.05 \pm 1.05$ & 0.965 & $-0.21 \pm 0.64$ & 0.742
\end{tabular}

Numbers are linear mixed-model coefficients \pm standard error, relative to the $A \beta$ - group, adjusted for age, gender and years of education $A \beta$ amyloid-beta, $A D$ Alzheimer's disease, $M C l$ mild cognitive impairment, MMSE Mini Mental State Examination, NC normal cognition decline at a faster rate than the $\mathrm{A} \beta$ - individuals $(\mathrm{NC}, p<$ 0.001 ; MCI, $p<0.001)$. For the demented subjects, $\mathrm{A} \beta$ did not influence the rate of decline (Table 4, Fig. 2).

\section{Discussion}

The aim of the EMIF consortium is to enable the reutilization of preexisting data including the finding and assessment of relevant datasets and facilitation of their interoperability and reuse. For the EMIF-AD component, a major use-case objective has been to use the processes and tools established in the consortium to generate a novel cross-cohort data and sample collection for the discovery and validation of biomarkers for use in clinical trials using a multimodal and endophenotype design. The first results presented in this report confirm the central roles of $A \beta$ and $A P O E \& 4$ in the pathogenesis of $\mathrm{AD}$, which is consistent with findings from other large cohorts [36, 37]. The molecular studies are ongoing and will be reported in future publications.

$\mathrm{AD}$ is a complex and multifactorial disorder, which underscores the need for multimodal studies with sufficient statistical power [38]. To date these large studies are scarce, especially those including subjects across the whole clinical AD spectrum. To our knowledge, the only other large-scale studies that collected plasma, DNA, CSF and imaging material from individuals in various cognitive stages are the Alzheimer's Disease Neuroimaging Initiative (ADNI) [36] and the Australian Imaging, Biomarkers and Lifestyle (AIBL) study of aging [37] studies. Since these datasets are so unique, findings from these studies are difficult to validate. The current study will not only be of great additional value due to its explorative nature and complementary laboaratory analyses, but also because previous findings can be validated in a large-size cohort with multimodal data. We collected a wide variety of clinical variables including neuropsychological tests, comorbidities, medication use and psychiatric questionnaires. All of the clinical data and results from the multimodal wet-lab analyses will be stored on an online, secure data platform (tranSMART). Research proposals can be submitted via the EMIF-AD Catalog (https://emif-catalogue.eu) to work with these data, which require approval from the EMIF-AD team and the data-owners.

Besides the major advantages, this study also has some limitations. Currently, we do not have clinical follow-up data for all subjects, as some centers are still in the process of collecting these. However, these data may be added to the database in the future. Also, the data, samples and scans contributed to this study were collected at different centers and were not collected using the same protocol, which will lead to preanalytical variability. To limit this variability, the samples were analyzed centrally and the clinical data were harmonized using standardized values and dichotomous variables. 


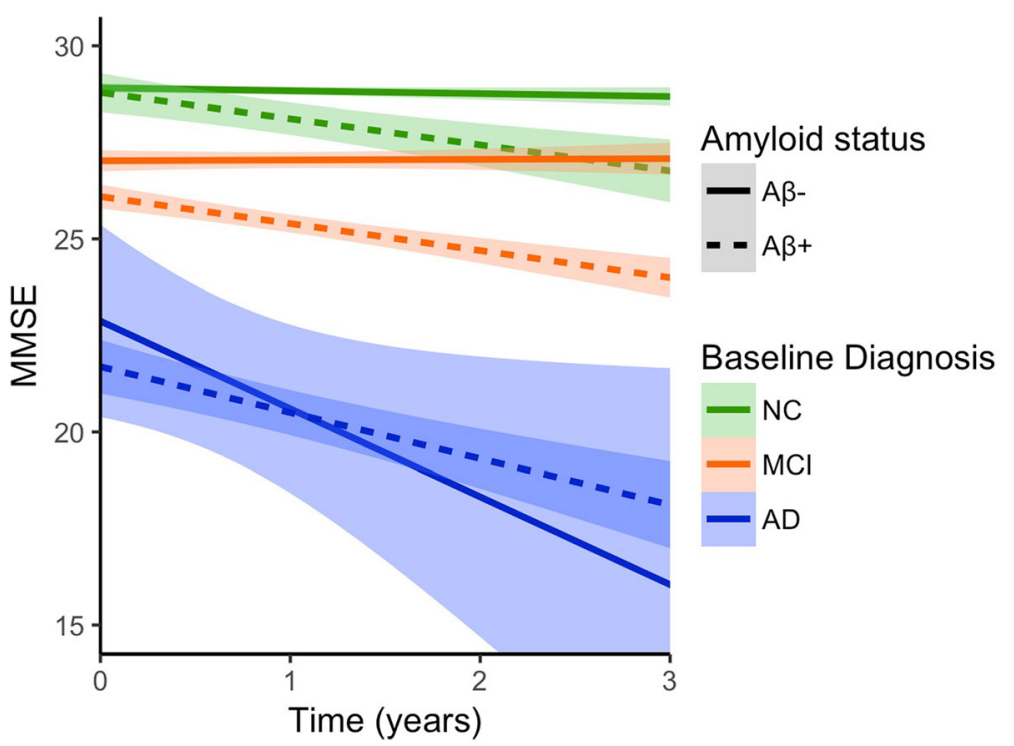

Fig. 2 MMSE scores over time by A $\beta$ status and diagnosis. Mean scores and $95 \%$ confidence intervals of MMSE over time for three diagnostic groups and by $A \beta$ status, adjusted for demographics. Solid lines represent $A \beta$ - groups, dashed lines represent $A \beta+$ groups. $A \beta$ amyloid-beta, $A D$ Alzheimer's disease, MCI mild cognitive impairment, MMSE Mini Mental State Examination, NC normal cognition

\section{Conclusion}

The various complementary analyses conducted in plasma, DNA and CSF and on MRI scans in a large-sized cohort of individuals across the clinical AD spectrum provide a unique opportunity to discover novel diagnostic and prognostic markers, and will also increase knowledge into the $\mathrm{AD}$ pathophysiology, which is required for the development of novel therapeutic interventions.

\section{Additional files}

Additional file 1: Table S1. Diagnostic criteria per cohort. Cohorts, countries, number of subjects and diagnostic criteria used for $\mathrm{NC}, \mathrm{MCl}$ and AD dementia (DOCX $90 \mathrm{~kb}$ )

Additional file 2: Table S2. Number of subjects per test by cognitive domain at baseline. Number of subjects per test and norms used in domains of global cognition, memory, language, attention, executive functioning and visuoconstruction (DOCX $90 \mathrm{~kb}$ )

Additional file 3: Table S3. Biomarker protocol information for each cohort. Protocol information for PET, CSF and plasma biomarker collection (DOCX $100 \mathrm{~kb}$ )

Additional file 4: Table S4. Ethical approval committee of each center. Ethical approval committees in each of the participating centers (DOCX $109 \mathrm{~kb})$

\section{Abbreviations}

AD: Alzheimer's disease; ADP: Affiliated data provider; APOE: Apolipoprotein E; Aß: Amyloid-beta; CSF: Cerebrospinal fluid; IMI: Innovative Medicine Initiative; MCl: Mild cognitive impairment; MMSE: Mini Mental State Examination; MRI: Magnetic resonance imaging; NC: Normal cognition; NFL: Neurofilament light; Ng: Neurogranin; PET: Positron emission tomography

\section{Acknowledgements}

The authors would like to thank all people involved in data and sample collection and/or logistics across the different centers, and in particular: Marije Benedictus, Wiesje van de Flier, Charlotte Teunissen, Ellen De Roeck, Naomi De Roeck, Ellis Niemantsverdriet, Charisse Somers, Babette Reijs, Andrea Izagirre Otaegi, Mirian Ecay Torres, Sindre Rolstad, Eva Bringman, Domilé Tautvydaité, Barbara Moullet, Charlotte Evenepoel, Isabelle Cleynen, Bea Bosch, Daniel Alcolea Rodriguez, Moira Marizzoni, Alberto Redolfi and Paolo Bosco.

\section{Funding}

The present study was conducted as part of the EMIF-AD project which has received support from the Innovative Medicines Initiative Joint Undertaking under EMIF grant agreement $n^{\circ} 115372$, the resources of which are composed of financial contribution from the European Union's Seventh Framework Program (FP7/2007-2013) and EFPIA companies' in kind contribution. The DESCRIPA study was funded by the European Commission within the 5th framework program (QLRT-2001-2455). The EDAR study was funded by the European Commission within the 5th framework program (contract \# 37670). The Leuven cohort was funded by the Stichting voor Alzheimer Onderzoek (grant numbers \#11020, \#13007 and \#15005). RV is a senior clinical investigator of the Flemish Research Foundation (FWO). The San Sebastian GAP study is partially funded by the Department of Health of the Basque Government (allocation 17.0.1.08.12.0000.2.454.01.41142.001.H). The authors acknowledge the contribution of the personnel of the Genomic Service Facility at the VIB-U Antwerp Center for Molecular Neurology. The research at VIB-CMN is funded in part by the University of Antwerp Research Fund.

\section{Availability of data and materials}

The dataset presented in the current study is available upon request and after approval of each data provider, via the EMIF-AD platform (https://emifcatalogue.eu; http://www.emif.eu/about/emif-ad).

\section{Authors' contributions}

IB contributed to study coordination, data management and statistical analyses, and wrote the manuscript. SV contributed to study coordination and wrote the manuscript. RV, PS, SE, GF, JLM, AW, AL, JP and PM-L contributed to data and sample collection. $\mathrm{AB}$ was responsible for plasma proteomic analyses. RD was responsible for multimodal analyses. CL-Q was responsible for plasma metabolomics analyses. KS and CVB were responsible for genetics (exome sequencing). LB was responsible for 
genetics (DNA extraction and QC, genome-wide SNP genotyping and methylation profiling). MtK and FB were responsible for MRI analyses. $\mathrm{HZ}$ was responsible for CSF analyses. SL and JS contributed to study design and study coordination. PJV contributed to study design and study coordination, and wrote the manuscript. All authors read and approved the final manuscript.

\section{Ethics approval and consent to participate}

Written informed consent was obtained from all participants before inclusion in the study. The medical ethics committee at each site approved the study (Additional file 4: Table S4).

\section{Competing interests}

The authors declare that they have no competing interests.

\section{Publisher's Note}

Springer Nature remains neutral with regard to jurisdictional claims in published maps and institutional affiliations.

\section{Author details \\ ${ }^{1}$ Alzheimer Centrum Limburg, Maastricht University, Maastricht, the} Netherlands. ${ }^{2}$ University Hospital Leuven, Leuven, Belgium. ${ }^{3}$ Laboratory for Cognitive Neurology, Department of Neurosciences, KU Leuven, Leuven, Belgium. ${ }^{4}$ Alzheimer Center and Department of Neurology, VU University Medical Center, Amsterdam, the Netherlands. ${ }^{5}$ Reference Center for Biological Markers of Dementia (BIODEM), University of Antwerp, Antwerp, Belgium. ${ }^{6}$ Department of Neurology and Memory Clinic, Hospital Network Antwerp (ZNA) Middelheim and Hoge Beuken, Antwerp, Belgium. 'University of Antwerp, Antwerp, Belgium. ${ }^{8}$ University of Geneva, Geneva, Switzerland. ${ }^{9}$ IRCCS Instituto Centro San Giovanni di Dio Fatebenefratelli, Brescia, Italy.

${ }^{10}$ Alzheimer's Disease \& Other Cognitive Disorders Unit, Hospital Clínic_IDIBAPS, Barcelona, Spain. ${ }^{11}$ Barcelona Beta Brain Research Center, Fundació Pasqual Maragall, Barcelona, Spain. ${ }^{12}$ Institute of Neuroscience and Physiology, Moelndal, Sweden. ${ }^{13}$ Hospital de la Santa Creu i Sant Pau, Barcelona, Spain. ${ }^{14}$ Geriatric Psychiatry, Department of Mental Health and Psychiatry, Geneva University Hospitals, Geneva, Switzerland. ${ }^{15}$ Department of Psychiatry, University Hospital of Lausanne, Lausanne, Switzerland. ${ }^{16}$ Center for Research and Advanced Therapies, CITA-Alzheimer Foundation, San Sebastian, Spain. ${ }^{17}$ University of Oxford, Oxford, UK. ${ }^{18}$ King's College London, London, UK. ${ }^{19} \mathrm{NIHR}$ Biomedical Research Centre for Mental Health and Biomedical Research Unit for Dementia at South London and Maudsley NHS Foundation, London, UK. ${ }^{20}$ Farr Institute of Health Informatics Research, UCL Institute of Health Informatics, University College London, London, UK. ${ }^{21}$ NIHR University College London Hospitals Biomedical Research Centre, London, UK. ${ }^{22}$ Neurodegenerative Brain Diseases Group, VIB-Department of Molecular Genetics, Antwerp, Belgium. ${ }^{23}$ Institute Born-Bunge, University of Antwerp, Antwerp, Belgium. ${ }^{24}$ Lübeck Interdisciplinary Platform for Genome Analytics, University of Lübeck, Lübeck, Germany. ${ }^{25}$ School of Public Health, Imperial College London, London, UK. ${ }^{26}$ Department of Psychology, University of Oslo, Oslo, Norway. ${ }^{27}$ Department of Radiology and Nuclear Medicine, VU University Medical Center, Amsterdam, the Netherlands. ${ }^{28}$ Department of Psychiatry and Neurochemistry, University of Gothenburg, Mölndal, Sweden. ${ }^{29}$ Clinical Neurochemistry Laboratory, Sahlgrenska University Hospital, Mölndal, Sweden. ${ }^{30}$ Department of Molecular Neuroscience, UCL Institute of Neurology, London, UK. ${ }^{31}$ UK Dementia Research Institute, London, UK. ${ }^{32}$ Experimental Medicine, Janssen Pharmaceutical Companies, Beerse, Belgium. ${ }^{33}$ Department of Psychiatry \& Neuropsychology, School for Mental Health and Neuroscience, Alzheimer Center Limburg, Maastricht University, Universiteitssingel 40, Box 34, P.O. Box 616,6200, MD, Maastricht, the Netherlands.

\section{Received: 10 October 2017 Accepted: 8 June 2018} Published online: 06 July 2018

\section{References}

1. Jack CR, Knopman DS, Jagust WJ, Petersen RC, Weiner MW, Aisen PS, Shaw LM, Vemuri P, Wiste HJ, Weigand SD, et al. Tracking pathophysiological processes in Alzheimer's disease: an updated hypothetical model of dynamic biomarkers. Lancet Neurol. 2013;12(2):207-16.
2. Bateman RJ, Xiong C, Benzinger TL, Fagan AM, Goate A, Fox NC, Marcus DS, Cairns NJ, Xie X, Blazey TM, et al. Clinical and biomarker changes in dominantly inherited Alzheimer's disease. N Engl J Med. 2012;367(9):795-804.

3. Sperling R, Mormino E, Johnson K. The evolution of preclinical Alzheimer's disease: implications for prevention trials. Neuron. 2014;84(3):608-22.

4. Mattsson N, Carrillo MC, Dean RA, Devous MD Sr, Nikolcheva T, Pesini P, Salter H, Potter WZ, Sperling RS, Bateman RJ, et al. Revolutionizing Alzheimer's disease and clinical trials through biomarkers. Alzheimers Dement (Amst). 2015;1(4):412-9.

5. Hampel H, Frank R, Broich K, Teipel SJ, Katz RG, Hardy J, Herholz K, Bokde AL, Jessen F, Hoessler YC, et al. Biomarkers for Alzheimer's disease: academic, industry and regulatory perspectives. Nat Rev Drug Discov. 2010;9(7):560-74.

6. Vandenberghe R, Adamczuk K, Dupont P, Laere KV, Chetelat G. Amyloid PET in clinical practice: its place in the multidimensional space of Alzheimer's disease. Neuroimage Clin. 2013;2:497-511.

7. Scheltens P, Blennow K, Breteler MM, de Strooper B, Frisoni GB, Salloway S, Van der Flier WM. Alzheimer's disease. Lancet. 2016;388(10043):505-17.

8. Duits FH, Prins ND, Lemstra AW, Pijnenburg YA, Bouwman FH, Teunissen CE, Scheltens P, van der Flier WM. Diagnostic impact of CSF biomarkers for Alzheimer's disease in a tertiary memory clinic. Alzheimers Dement. 2015;11(5):523-32.

9. Rathore $S$, Habes M, Iftikhar MA, Shacklett A, Davatzikos C. A review on neuroimaging-based classification studies and associated feature extraction methods for Alzheimer's disease and its prodromal stages. Neurolmage. 2017;155:530-48.

10. Laske C. Blood-based biomarkers in Alzheimer disease: where are we now and where have we to go? JAMA Neurol. 2013;70(1):133.

11. Snyder HM, Carrillo MC, Grodstein F, Henriksen K, Jeromin A, Lovestone S, Mielke MM, O'Bryant S, Sarasa M, Sjogren M, et al. Developing novel blood-based biomarkers for Alzheimer's disease. Alzheimers Dement. 2014;10(1):109-14.

12. Galasko D, Golde TE. Biomarkers for Alzheimer's disease in plasma, serum and blood-conceptual and practical problems. Alzheimers Res Ther. 2013;5(2):10

13. Baird AL, Westwood S, Lovestone S. Blood-based proteomic biomarkers of Alzheimer's disease pathology. Front Neurol. 2015;6:236.

14. Reijs BLR, Ramakers I, Elias-Sonnenschein L, Teunissen CE, Koel-Simmelink M, Tsolaki M, Wahlund LO, Waldemar G, Hausner L, Johannsen P, et al. Relation of odor identification with Alzheimer's disease markers in cerebrospinal fluid and cognition. J Alzheimers Dis. 2017;60(3):1025-34.

15. Galluzzi S, Marizzoni M, Babiloni C, Albani D, Antelmi L, Bagnoli C, BartresFaz D, Cordone S, Didic M, Farotti L, et al. Clinical and biomarker profiling of prodromal Alzheimer's disease in workpackage 5 of the Innovative Medicines Initiative PharmaCog project: a 'European ADNI study. J Intern Med. 2016;279(6):576-91.

16. Visser PJ, Verhey FR, Boada M, Bullock R, De Deyn PP, Frisoni GB, Frolich L, Hampel H, Jolles J, Jones R, et al. Development of screening guidelines and clinical criteria for predementia Alzheimer's disease. The DESCRIPA study. Neuroepidemiology. 2008;30(4):254-65.

17. Somers C, Struyfs H, Goossens J, Niemantsverdriet E, Luyckx J, De Roeck N, De Roeck E, De Vil B, Cras P, Martin JJ, et al. A decade of cerebrospinal fluid biomarkers for Alzheimer's disease in Belgium. J Alzheimers Dis. 2016;54(1):383-95.

18. van der Flier WM, Pijnenburg YA, Prins N, Lemstra AW, Bouwman FH, Teunissen CE, van Berckel BN, Stam CJ, Barkhof F, Visser PJ, et al. Optimizing patient care and research: the Amsterdam Dementia Cohort. J Alzheimers Dis. 2014;41(1):313-27.

19. Alcolea D, Carmona-Iragui M, Suarez-Calvet M, Sanchez-Saudinos MB, Sala I, Anton-Aguirre S, Blesa R, Clarimon J, Fortea J, Lleo A. Relationship between beta-Secretase, inflammation and core cerebrospinal fluid biomarkers for Alzheimer's disease. J Alzheimers Dis. 2014;42(1):157-67.

20. Fortea J, Sala-Llonch R, Bartres-Faz D, Bosch B, Llado A, Bargallo N, Molinuevo $J$, Sanchez-Valle R. Increased cortical thickness and caudate volume precede atrophy in PSEN1 mutation carriers. J Alzheimers Dis. 2010;22(3):909-22.

21. Adamczuk K, Schaeverbeke J, Nelissen N, Neyens V, Vandenbulcke M, Goffin K, Lilja J, Hilven K, Dupont P, Van Laere K, et al. Amyloid imaging in cognitively normal older adults: comparison between (18)Fflutemetamol and (11)C-Pittsburgh compound B. Eur J Nucl Med Mol Imaging. 2016;43(1):142-51. 
22. Estanga A, Ecay-Torres M, Ibanez A, Izagirre A, Villanua J, Garcia-Sebastian M, Iglesias Gaspar MT, Otaegui-Arrazola A, Iriondo A, Clerigue M, et al. Beneficial effect of bilingualism on Alzheimer's disease CSF biomarkers and cognition. Neurobiol Aging. 2017;50:144-51.

23. Wallin A, Nordlund A, Jonsson M, Blennow K, Zetterberg H, Ohrfelt A, Stalhammar J, Eckerstrom M, Carlsson M, Olsson E, et al. Alzheimer's disease - subcortical vascular disease spectrum in a hospital-based setting: overview of results from the Gothenburg $\mathrm{MCl}$ and dementia studies. J Cereb Blood Flow Metab. 2016;36(1):95-113.

24. Tautvydaite D, Kukreja D, Antonietti JP, Henry H, von Gunten A, Popp J. Interaction between personality traits and cerebrospinal fluid biomarkers of Alzheimer's disease pathology modulates cognitive performance. Alzheimers Res Ther. 2017;9(1):6.

25. Morris JC. The Clinical Dementia Rating (CDR): current version and scoring rules. Neurology. 1993:43(11):2412-4.

26. Reisberg B, Ferris SH, de Leon MJ, Crook T. The Global Deterioration Scale for assessment of primary degenerative dementia. Am J Psychiatry. 1982;139(9):1136-9.

27. Petersen RC. Mild cognitive impairment as a diagnostic entity. J Intern Med. 2004;256(3):183-94.

28. Winblad B, Palmer K, Kivipelto M, Jelic V, Fratiglioni L, Wahlund LO, Nordberg A, Backman L, Albert M, Almkvist O, et al. Mild cognitive impairment-beyond controversies, towards a consensus: report of the International Working Group on Mild Cognitive Impairment. J Intern Med. 2004;256(3):240-6.

29. McKhann G, Drachman D, Folstein M, Katzman R, Price D, Stadlan EM. Clinical diagnosis of Alzheimer's disease: report of the NINCDS-ADRDA work group under the auspices of Department of Health and Human Services Task Force on Alzheimer's disease. Neurology. 1984;34(7):939-44.

30. Scheufele E, Aronzon D, Coopersmith R, McDuffie MT, Kapoor M, Uhrich CA, Avitabile JE, Liu J, Housman D, Palchuk MB. tranSMART: an open source knowledge management and high content data analytics platform. AMIA Jt Summits Transl Sci Proc. 2014:2014:96-101.

31. Hye A, Riddoch-Contreras J, Baird AL, Ashton NJ, Bazenet C, Leung R, Westman E, Simmons A, Dobson R, Sattlecker M, et al. Plasma proteins predict conversion to dementia from prodromal disease. Alzheimers Dement. 2014:10(6):799-807. e792

32. Scheltens $P$, Leys $D$, Barkhof $F$, Huglo D, Weinstein HC, Vermersch $P$, Kuiper M, Steinling M, Wolters EC, Valk J. Atrophy of medial temporal lobes on MRI in "probable" Alzheimer's disease and normal ageing: diagnostic value and neuropsychological correlates. J Neurol Neurosurg Psychiatry. 1992;55(10):967-72.

33. Pasquier F, Leys D, Weerts JG, Mounier-Vehier F, Barkhof F, Scheltens P. Inter- and intraobserver reproducibility of cerebral atrophy assessment on MRI scans with hemispheric infarcts. Eur Neurol. 1996;36(5):268-72.

34. Koedam EL, Lehmann M, van der Flier WM, Scheltens P, Pijnenburg YA, Fox N, Barkhof F, Wattjes MP. Visual assessment of posterior atrophy development of a MRI rating scale. Eur Radiol. 2011:21(12):2618-25.

35. Redolfi A, Bosco P, Manset D, Frisoni GB. Brain investigation and brain conceptualization. Funct Neurol. 2013;28(3):175-90.

36. Mueller SG, Weiner MW, Thal $\amalg$, Petersen RC, Jack C, Jagust W, Trojanowski JQ, Toga AW, Beckett L. The Alzheimer's Disease Neuroimaging Initiative. Neuroimaging Clin N Am. 2005;15(4):869-77. xi-xii.

37. Ellis KA, Bush Al, Darby D, De Fazio D, Foster J, Hudson P, Lautenschlager NT, Lenzo N, Martins RN, Maruff P, et al. The Australian Imaging, Biomarkers and Lifestyle (AIBL) study of aging: methodology and baseline characteristics of 1112 individuals recruited for a longitudinal study of Alzheimer's disease. Int Psychogeriatr. 2009;21 (4):672-87.

38. Perrin RJ, Fagan AM, Holtzman DM. Multimodal techniques for diagnosis and prognosis of Alzheimer's disease. Nature. 2009;461(7266):916-22.

Ready to submit your research? Choose BMC and benefit from:

- fast, convenient online submission

- thorough peer review by experienced researchers in your field

- rapid publication on acceptance

- support for research data, including large and complex data types

- gold Open Access which fosters wider collaboration and increased citations

- maximum visibility for your research: over $100 \mathrm{M}$ website views per year

At BMC, research is always in progress.

Learn more biomedcentral.com/submissions 